\title{
Kommunalpolitik in Deutschland: kohärenter, informativer Sammelband
}

\begin{abstract}
Kost, Andreas und Hans-Georg Wehling (Hrsg.): Kommunalpolitik in den deutschen Ländern. Eine Einführung, 2. aktualisierte und überarbeitete Auflage, VS Verlag für Sozialwissenschaften, Wiesbaden 2010, 413 Seiten, € 34,95.
\end{abstract}

In den letzten zwei Jahrzehnten gab es auf kommunaler Ebene umfassende institutionelle Reformprozesse, die dazu geführt haben, dass sich die „Rahmenbedingungen von Kommunalpolitik (...) grundlegend verändert" (S. 13) haben, wie die Herausgeber Hans-Georg Wehling und Andreas Kost in ihrer Einleitung konstatieren. Einerseits werden die Bürgermeister mittlerweile in allen Bundesländern direkt gewählt und sind, aufgrund der fast flächendeckenden Einführung von Variationen der süddeutschen Ratsverfassung, zudem mit größeren Kompetenzen ausgestattet. Andererseits wurden direktdemokratische Beteiligungsmöglichkeiten auf kommunaler Ebene ausgeweitet. Diese Reformprozesse rückt der unter der Ägide der Landeszentralen für politische Bildung entstandene Sammelband in einer zweiten aktualisierten und überarbeiteten Auflage in den Blick. Ziel der als Lehrbuch und Einführung konzipierten Publikation ist dabei neben einer systematischen Bestandsaufnahme der „rechtlichen, ,nachlesbaren“ Veränderungen“ (S. 10) der Kommunalverfassungssysteme in den einzelnen Bundesländern auch die Analyse ihrer Auswirkungen.

Der Band gliedert sich in zwei große Teilbereiche: In sechzehn Einzelbeiträgen werden zunächst Charakteristika und Eigenheiten der kommunalen Entscheidungssysteme in den deutschen Bundesländern vor dem Hintergrund der in den vergangenen zwanzig Jahren durchgeführten Reformprozesse dargestellt. Im zweiten Teil werden in vergleichender Perspektive drei Themenkomplexe, die für die aktuelle kommunale Politik von wesentlicher Bedeutung sind, ausführlich analysiert und diskutiert; erstens die Veränderungen im kommunalpolitischen Entscheidungssystem im Hinblick auf die Stellung des Rates und des Bürgermeisters, zweitens die Einbindung der Kommunen in die bundesdeutsche Finanzverfassung und drittens die Bedeutung direktdemokratischer Entscheidungsstrukturen auf kommunaler Ebene.

Einführend rekapitulieren Hans-Georg Wehling und Andreas Kost die - inzwischen durch die Reformprozesse überholte - institutionelle Ausgestaltung der Kommunen Anfang der 1990er Jahre. Die knappe und aufs Wesentliche konzentrierte Einleitung legt damit ein Fundament für die Einordnung der beiden grundlegenden Reformprozesse, die Direktwahl der Bürgermeister und die Ausweitung von direktdemokratischen Beteiligungsformen. Beide Entwicklungen führen zu einer Verschiebung des Machtverhältnisses zwischen Bürgermeister und Gemeinderat und beeinflussen mithin die Strukturen kommunaler Entscheidungsprozesse in vielen Bundesländern. Vor diesem Hintergrund begleitet die vorgenommene Aktualisierung des im Jahr 2003 erstmals erschienenen Sammelbandes noch stattfindende Veränderungsprozesse und bietet eine notwendige grundlegende Bestandsaufnahme der einzelnen Kommunalsysteme in den Bundesländern zum jetzigen Zeitpunkt. Eine Stärke des Sammelbandes liegt denn auch darin, dass es den Herausgebern für diese Auflage noch stärker gelungen ist, die Autoren auf eine gemeinsame Linie der Länderdarstellung zu verpflichten. Dies sorgt im Vergleich zur ersten Auflage insgesamt für eine größere Kohärenz, die für den Leser - gerade im Vergleich der einzelnen Kommunalsysteme - gewinnbringend ist. So beginnt jeder Beitrag mit einem Überblick zur landesspezifischen Selbstverwaltungstradition, bevor schwerpunktmäßig die Veränderungen der institu- 
tionellen Ausgestaltung des Verhältnisses von Rat und Bürgermeister auf Gemeinde- und Kreisebene dargestellt und in ihren Wirkungen für den jeweiligen Landeskontext erläutert werden. Hieran schließt bei den meisten Beiträgen ein Abriss zu Wahlen und Wahlsystemen an, bevor Möglichkeiten der Bürgerbeteiligung und der direkten Demokratie auf kommunaler Ebene beschrieben werden.

Innerhalb dieser gemeinsamen Leitthemen setzen die sechzehn Autoren der Länderbeiträge jedoch durchaus eigene Akzente. Beispielsweise konzentriert sich der Aufsatz von Andreas Kost zu Nordrhein-Westfalen stark auf das Thema direkte Bürgerbeteiligung, und Michael Scherer informiert zusätzlich umfangreich über die Veränderung des Wahlrechts im Land Bremen. Das Kommunalsystem im Bundesland Sachsen-Anhalt, in der ersten Auflage des Sammelbandes auf nur vier Seiten behandelt, wird von der neuen Autorin, Marion Reiser, breiter dargestellt. Kenntnisreich führt sie die einzelnen Leitthemen aus und nimmt zudem verstärkt auf die sachsen-anhaltinischen Kommunalwahlen Bezug. Insgesamt ist der erste Teilbereich durch die detaillierten Beschreibungen der institutionellen Ausgestaltung der Kommunalwahlsysteme durch Landesexperten und durch die, hier nur an einigen Beispielen illustrierte, Varianz innerhalb der einzelnen Beiträge sowohl für Novizen als auch für Experten informativ und lohnend.

Ergänzend werden im zweiten Teilbereich drei ausgewählte Themenbereiche der Kommunalpolitik näher betrachtet: Hans-Georg Wehling fragt in seinem lesenswerten Aufsatz nach Widerständen gegen die Reformprozesse und nach ihren Auswirkungen auf die Machtverteilung zwischen Bürgermeister und Rat. Sein Fazit: die Reformprozesse haben zu einer doppelten „Entmachtung“ (S. 365) des Rates geführt, eine Entwicklung, die er im Hinblick auf den dadurch beschränkten Einfluss von Parteien in der Kommunalpolitik durchaus positiv bewertet. Hiermit werde anerkannt, dass „Kommunalpolitik eher Verwaltung ist, pragmatisch an den lokalen Problemen ausgerichtet und nicht so sehr Politik“ (S. 365).

Wolfgang Scherf stellt im Anschluss detailliert die Grundlagen der föderalen Finanzverfassung dar, skizziert die Stellung der Kommunen darin und zeigt Probleme und Reformansätze auf. Er macht deutlich, dass die konjunkturabhängige Instabilität das Hauptproblem des kommunalen Finanzsystems ist und dass dieses nur über grundlegende Reformen gelöst werden kann. Scherf berührt die „Gretchenfrage“ kommunaler Politik: Können formale politische Handlungsspielräume überhaupt genutzt werden, wenn es insgesamt an Geld mangelt? Leider versäumt er an dieser Stelle, einen Zusammenhang herzustellen zwischen der kommunalen Finanzkrise und der lokalen Demokratie im Allgemeinen sowie der direkten Demokratie im Besonderen. Letztere untersucht Andreas Kost im abschließenden Beitrag auf ihre tatsächliche Nutzung und ihre Effekte auf der lokalen Ebene. Er hebt besonders die antizipatorische Wirkung plebiszitärer Instrumente auf die politischen Entscheidungsträger hervor und plädiert für ihre Ausweitung.

Insgesamt bleibt festzuhalten, dass der in sich ungewöhnlich kohärente Sammelband einen grundlegenden Einstieg und profunden Überblick zu den einzelnen Kommunalsystemen sowie zu ausgewählten Themenbereichen bietet und damit seinem Einführungs- und Lehrbuchcharakter mehr als gerecht wird. Besonders für Studierende erweist sich zudem der Anhang als nützlich, der das „Kommunale Wahlrecht im Ländervergleich“ und die Bürgermeisterwahlen in deutschen Ländern“ (S. 406) summarisch in Tabellenform darstellt und zudem mit einem kurzen kommentierten Literaturverzeichnis aktueller Bestandsaufnahmen der Kommunalpolitik aufwartet. 